\title{
Narrow banded wave propagation from very deep waters to the shore
}

\author{
Gonzalo Simarro* \\ ICM (CSIC), Passeig Maritim de la Barceloneta 37-49, 08003 Barcelona, Spain \\ Alvaro Galan \\ ETSICCP (UCLM), Avenida Camilo Jose Cela 2, 13071 Ciudad Real, Spain \\ Roberto Minguez \\ IH Cantabria (UC), Calle Isabel Torres 15, 39011 Santander, Spain \\ Alejandro Orfila \\ IMEDEA (CSIC-UIB), Carrer Miquel Marques 21, 07190 Esporles, Spain
}

\section{Abstract}

A fully nonlinear Boussinessq-type model with several free coefficients is considered as a departure point. The model is monolayer and low order so as to simplify numerical solvability. The coefficients of the model are here considered functions of the local water depth. In doing so, we allow to improve the dispersive and shoaling properties for narrow banded wave trains in very deep waters. In particular, for monochromatic waves the dispersion and shoaling errors are bounded by $\simeq 2.8 \%$ up to $k h=100$, being $k$ the wave number and $h$ the water depth. The proposed model is fully nonlinear in weakly dispersive conditions, so that nonlinear wave decomposition in shallower waters is

\footnotetext{
*corresponding author

Email address: simarro@icm.csic.es (Gonzalo Simarro)
} 
well reproduced. The model equations are numerically solved using a fourth order scheme and tested against analytical solutions and experimental data. Keywords: Phase-resolving wave propagation models, Boussinesq-type equations, linear dispersion and shoaling, numerical schemes.

\section{Introduction}

In deep waters water wave propagation does not depend on water depth. For instance, the wave celerity $c$ for a wave with period $\mathrm{T}$ is $c=g \mathrm{~T} / 2 \pi$, with $g$ the gravity acceleration. Because each wave period, T, travels with a different velocity, deep waters are called dispersive. Furthermore, in deep waters the wave amplitude, $a$, is usually much smaller than the water depth $h$ and, as a consequence, the model equations are linear (Airy theory).

As the water waves propagate to the coast, the water depth $h$ decreases and the wave propagation becomes influenced by it. Also, nonlinear effects become important. In shallow waters, where the wave propagation is dominated by the water depth, the wave celerity is given by $c \approx \sqrt{g h}$, which is independent of the wave period (i.e., non dispersive). An important physical property of shallow waters is that the horizontal velocity profile is nearly uniform in the vertical. Nonlinear Shallow Waters Equations (NSWEs), which are vertically integrated, exploit this property and are valid for non dispersive conditions and for arbitrary amplitudes of the wave.

It is accepted that shallow water conditions correspond to $k h \lesssim 0.3$, with $k=2 \pi / \lambda$ the wave number and $\lambda$ the wave length, while $k h \gtrsim 3$ corresponds to deep waters (Dean and Dalrymple, 1984). In intermediate waters (namely $0.3 \lesssim k h \lesssim 3)$ nonlinearity and dispersion coexist and neither Airy theory 
nor NSWE can represent the physics. To overcome this problem, two main pertubation approaches are found (Dingemans, 1997). On the one hand, Stokes theory departs from the fully dispersive linear Airy theory to incorporate weakly nonlinear effects. On the other, Boussinesq-Type Equations (BTEs) depart from NSWEs and include weakly dispersive effects. This work is focused on BTEs.

Being $a_{0}, h_{0}$ and $k_{0}$ characteristic values for wave amplitude, water depth and wave number respectively, the dimensionless parameters

$$
\epsilon \equiv \frac{a_{0}}{h_{0}}, \quad \text { and } \quad \mu \equiv k_{0} h_{0},
$$

represent nonlinear and dispersive effects respectively. The NSWEs can represent fully nonlinear waves for the nondispersive case. The original BTEs by Peregrine (1967) included all the nonlinear non dispersive terms (NSWEs) plus the weakly nonlinear and weakly dispersive terms $\mathcal{O}\left(\epsilon^{1} \mu^{2}\right)$, but disregarded the highly nonlinear and weakly dispersive terms $\mathcal{O}\left(\epsilon^{2} \mu^{2}, \epsilon^{3} \mu^{2}\right)$. The inclusion of the highly nonlinear and weakly dispersive terms $\mathcal{O}\left(\epsilon^{2} \mu^{2}, \epsilon^{3} \mu^{2}\right)$ was done, e.g., by Green and Naghdi (1976) and Wei et al. (1995).

The equations by Peregrine (1967) were derived for the depth averaged horizontal velocity, $\overline{\mathbf{u}}$, and give good linear dispersive performance, i.e., errors below $1 \%$ relative to Airy's celerity, up to $k h \lesssim 1.1$. To improve the range of applicability, several approaches are found in the literature. Two of them are higher order and multilayer models: higher order models include terms $\mathcal{O}\left(\mu^{4}\right)$ or higher (Gobbi et al., 2000), while the multilayer models split the flow into several layers, applying low order models into each one (Lynett and Liu, 2004). These two kind of models increase the numerical complexity for 
they include fifth order derivatives or more unknowns.

Based on the method of Agnon et al. (1999), Madsen et al. (2002) developed a fully nonlinear model, which is accurate in very deep water $(k h \lesssim 40$ for the linear case). Their model requires more differential equations to be solved, compared to other higher order models such as that by Gobbi et al. (2000), and the highest order of derivatives in the model is also fifth. Madsen et al. (2003) presented a simplified version of their original model, where the highest order of derivatives is reduced to three. The range of application is also reduced to $k h \lesssim 10$.

Using a low order monolayer model, Nwogu (1993) improved the linear dispersive performance up to $k h \lesssim 3.3$ by using the horizontal velocity $\mathbf{u}_{\alpha}$ at $z=z_{\alpha}$ instead of the depth averaged velocity proposed by Peregrine (1967). In fact, the above mentioned models by Wei et al. (1995) and Lynett and Liu (2004), amongst other, follow this idea to improve the linear dispersion performance.

Following the track of low order monolayer BTEs, Madsen and Schaffer (1998) modified the model equations by Wei et al. (1995) by introducing new terms which included free coefficients. While the equations remain exact up to $\mathcal{O}\left(\mu^{2}\right)$, similar to those by Wei et al. (1995), for the proposed coefficients they obtained errors in linear dispersion below $1 \%$ for $k h \lesssim 6.2$.

Although the improvements in linear dispersion, i.e., in the representation of the wave celerity, provided by Nwogu (1993) or Madsen and Schaffer (1998) are substantial, it is not generally so with the linear shoaling, i.e., with the representation of the wave amplitude: the linear shoaling by the equations by Nwogu (1993) and Madsen and Schaffer (1998) is fair (1\% error in wave 
amplitude) only up to $k h \approx 0.78$ and $k h \approx 0.82$ respectively, and at $k h=2$ the errors are already above $7.4 \%$ in both cases.

Other approaches have considered the improvement of linear properties to arbitrary depths which are to mention. Beji and Nadaoka (1999) followed a different approach also for narrow banded wave trains, whereas Karambas and Memos (2009) reached fully dispersion (i.e., for arbitrary depths and arbitrary ranges of frequencies). In both cases, however, the models do not allow fully nonlinearities in weakly dispersive conditions.

Departing from Madsen and Schaffer's equations and using an optimization approach, Galan et al. (2012) reduced the errors to $0.3 \%$ both in linear dispersion and shoaling up to $k h \approx 5$. Further, Galan et al. (2012) equations also include new terms $\mathcal{O}\left(\epsilon^{1} \mu^{4}\right)$ to improve the weakly nonlinear and highly dispersive properties.

All the above works consider that the free coefficients introduced are constant. In the present work we consider that these coefficients are functions of the water depth. In this way we will be able to improve the model properties up to deeper waters. As a counterpart, we will require that the wave train travelling to the coast is, in deep waters, narrow banded in frequencies. Narrow banded wave trains are associated to long fetchs (swells), and hence its usefulness. Further, in weakly dispersive conditions the equations will remain fully nonlinear.

This work can be considered as an extension of that by Lee et al. (2003) for the propagation of monochromatic waves in deep waters. The work by Lee et al. departed from Wei et al. (1995) equations, having only one free parameter and only monochromatic waves could be represented (strickly speaking, 
94

95

96

97

98

99

$$
\begin{aligned}
& \mathbf{Z}-\mathbf{Z}_{*}+ c_{1 \alpha} h \boldsymbol{\nabla} \boldsymbol{\nabla} \cdot(h \mathbf{Z})+c_{2 \alpha} h^{2} \boldsymbol{\nabla} \boldsymbol{\nabla} \cdot \mathbf{Z}-\boldsymbol{\nabla}\left[\eta \boldsymbol{\nabla} \cdot(h \mathbf{Z})+\frac{\eta^{2}}{2} \boldsymbol{\nabla} \cdot \mathbf{Z}\right] \\
&+\boldsymbol{\nabla} {\left[\left(c_{1 \alpha} h-\eta\right) \mathbf{u} \cdot \boldsymbol{\nabla X}+\left(c_{2 \alpha} h^{2}-\frac{\eta^{2}}{2}\right) \mathbf{u} \cdot \boldsymbol{\nabla} \mathrm{Y}+\frac{(\mathrm{X}+\eta \mathrm{Y})^{2}}{2}\right] } \\
&+\left(\gamma-\gamma_{h}\right) h^{2} \boldsymbol{\nabla} \boldsymbol{\nabla} \cdot\left(\mathbf{Z}-\mathbf{Z}_{*}\right)+\gamma_{h} h \boldsymbol{\nabla} \boldsymbol{\nabla} \cdot\left(h\left(\mathbf{Z}-\mathbf{Z}_{*}\right)\right) \\
&-\gamma_{\epsilon} \boldsymbol{\nabla}\left[\eta \boldsymbol{\nabla} \cdot\left(h\left(\mathbf{Z}-\mathbf{Z}_{*}\right)\right)\right]=0
\end{aligned}
$$

\section{Governing equations}

$$
\begin{aligned}
\mathrm{X}-\mathrm{X}_{*}+\boldsymbol{\nabla} & \cdot\left[d_{1 \alpha} h^{2} \boldsymbol{\nabla X}+d_{2 \alpha} h^{3} \nabla \mathrm{Y}\right] \\
+ & \boldsymbol{\nabla} \cdot\left[\left(c_{1 \alpha} h-\frac{\eta}{2}\right) \eta \boldsymbol{\nabla X}+\left(c_{2 \alpha} h^{2}-\frac{\eta^{2}}{6}\right) \eta \nabla \mathrm{Y}\right] \\
+ & \left(\delta-\delta_{h}\right) \boldsymbol{\nabla} \cdot\left[h^{2} \boldsymbol{\nabla}\left(\mathrm{X}-\mathrm{X}_{*}\right)\right]+\delta_{h} \nabla^{2}\left[h^{2}\left(\mathrm{X}-\mathrm{X}_{*}\right)\right] \\
& +\delta_{\epsilon} \boldsymbol{\nabla} \cdot\left[h \eta \boldsymbol{\nabla}\left(\mathrm{X}-\mathrm{X}_{*}\right)\right]=0,
\end{aligned}
$$

and
100

101

with $\eta$ the free surface elevation, $\mathbf{u}$ the horizontal velocity evaluated at $z=$ $z_{\alpha}=\alpha h, \mathrm{Y} \equiv \boldsymbol{\nabla} \cdot \mathbf{u}$ and 


\begin{tabular}{lrrr}
\hline \hline & W95 & M98 & G12 \\
\hline$\alpha$ & -0.53096 & -0.54122 & -0.54217 \\
$\delta$ & - & -0.03917 & -0.02409 \\
$\gamma$ & - & -0.01052 & -0.00492 \\
$\delta_{h}$ & - & -0.14453 & -0.15530 \\
$\gamma_{h}$ & - & -0.02153 & -0.07897 \\
$\delta_{\epsilon}$ & - & - & -0.36052 \\
$\gamma_{\epsilon}$ & - & - & 0.13169 \\
\hline \hline
\end{tabular}

Table 1: Constant coefficients for Wei et al. (1995), Madsen and Schaffer (1998) and Galan et al. (2012), denoted respectively as W95, M98 and G12.

$$
\begin{aligned}
\mathrm{X} & \equiv \boldsymbol{\nabla} \cdot(h \mathbf{u}), & \mathbf{Z} & \equiv \mathbf{u}_{t}, \\
\mathrm{X}_{*} & \equiv-\eta_{t}-\boldsymbol{\nabla} \cdot(\eta \mathbf{u}), & \mathbf{Z}_{*} & \equiv-\frac{1}{2} \boldsymbol{\nabla}(\mathbf{u} \cdot \mathbf{u})-g \boldsymbol{\nabla} \eta,
\end{aligned}
$$

with $g$ is the gravity acceleration. In equations (2)

$$
c_{1 \alpha} \equiv \alpha, \quad c_{2 \alpha} \equiv \frac{\alpha^{2}}{2}, \quad d_{1 \alpha} \equiv \alpha+\frac{1}{2}, \quad d_{2 \alpha} \equiv \frac{\alpha^{2}}{2}-\frac{1}{6},
$$

where $\alpha$ is a free coefficient, as well as $\delta, \gamma, \delta_{h}, \gamma_{h}, \delta_{\epsilon}$ and $\gamma_{\epsilon}$. Table 1 shows the values by Galan et al. (2012), hereafter "G12", and also the ones required to recover the equations by Madsen and Schaffer (1998) and Wei et al. (1995).

The equations (2) are obtained using an asymptotic expansion in $k h$ and are exact up to $\mathcal{O}\left((k h)^{2}\right)$. No limitations are imposed on the nonlinearity, so that they can represent fully nonlinear waves up to order $\mathcal{O}\left((k h)^{2}\right)$. For $k h \rightarrow 0$ they become independent of the coefficients and tend to the exact 
shallow water equations. The weighting coefficients influence the behavior of the equations only in deeper waters. Being more specific, the linear dispersion is influenced only by $\alpha, \delta$ and $\gamma$, coefficients $\delta_{h}$ and $\gamma_{h}$ only influence the linear shoaling and the coefficients $\delta_{\epsilon}$ and $\gamma_{\epsilon}$ affect only the nonlinear performance. All seven coefficients have been chosen so as to improve the linear and weakly nonlinear performance in deeper waters.

As shown by G12 for constant coefficients, the linear dispersion relationship embedded in the above equations (2) is

$$
\left\{\frac{c_{\mathrm{bte}}^{2}}{g h}=\right\} \frac{\omega^{2}}{g k_{\mathrm{bte}}^{2} h}=\frac{1-\left(d_{\alpha}+\gamma+\delta\right)\left(k_{\mathrm{bte}} h\right)^{2}+\left(d_{\alpha}+\delta\right) \gamma\left(k_{\mathrm{bte}} h\right)^{4}}{1-\left(c_{\alpha}+\gamma+\delta\right)\left(k_{\mathrm{bte}} h\right)^{2}+\left(c_{\alpha}+\gamma\right) \delta\left(k_{\mathrm{bte}} h\right)^{4}}
$$

where $c_{\mathrm{bte}}$ wave celetiry corresponding to these BTEs, $k_{\mathrm{bte}}$ the corresponding wave number, $\omega$ the wave angular frequency, $c_{\alpha} \equiv c_{\alpha, 1}+c_{\alpha, 2}=\alpha^{2} / 2+\alpha$ and $d_{\alpha} \equiv d_{\alpha, 1}+d_{\alpha, 2}$. The exact Airy dispersion expression is

$$
\left\{\frac{c_{\text {Airy }}^{2}}{g h}=\right\} \frac{\omega^{2}}{g k_{\text {Airy }}^{2} h}=\frac{\tanh \left(k_{\text {Airy }} h\right)}{k_{\text {Airy }} h} .
$$

For given values of gravity acceleration $g$, water depth $h$, wave angular frequency $\omega$ and the three coefficients $\alpha, \delta$ and $\gamma$, the values of $k_{\text {bte }}$ and $k_{\text {Airy }}$ obtained from the equations (5) and (6) are different in general, thus giving an error in the wave celerity (linear dispersion). Figure 1 shows the error in linear dispersion, defined as

$$
\varepsilon_{c} \equiv \frac{c_{\text {bte }}}{c_{\text {Airy }}}-1\left\{=\frac{k_{\text {Airy }}}{k_{\text {bte }}}-1\right\},
$$

as a function of the dimensionless group $\kappa \equiv \omega^{2} h / g$. This group, $\kappa$, can be used as a $k$-independent alternative to $\xi \equiv k h$ to evaluate whether deep or 
shallow waters hold (Nwogu, 1993). It has the advantage of not introducing $k$, which is different depending on whether equation (5) or (6) are used. For Airy theory $\kappa=\xi \tanh \xi$, and therefore, $\kappa \approx \xi$ for $\xi \gtrsim 3$.

Figure 1: Errors $\varepsilon_{c}$ and $\varepsilon_{s}$ for G12 (full lines), M98 (dashed) and W95 (dash-dotted). Shoaling errors, $\varepsilon_{s}$, are denoted with symbols.

Figure 1 also includes the error in the representation of wave amplitude assuming mild slope conditions. This error is defined as (Chen and Liu, 1995)

$$
\varepsilon_{s} \equiv \exp \left(\int_{0}^{h} \frac{\alpha_{\eta, \text { Airy }}-\alpha_{\eta, \text { bte }}}{h_{*}} d h_{*}\right)-1
$$

where $\alpha_{\eta, \text { Airy }}\left(\kappa_{*} \equiv \omega^{2} h_{*} / g\right)$ and $\alpha_{\eta, \text { bte }}\left(\kappa_{*}, \alpha, \delta, \gamma, \delta_{h}, \gamma_{h}\right)$ are the shoaling gradients for Airy's and above BTEs (Galan et al., 2012; Madsen and Sorensen, 1992). The error above defined is the relative error in the wave amplitude for a linear propagation over mild slopes from $\kappa$ to the shore, and it has been shown to be the proper error to be used (Chen and Liu, 1995; Lee et al., 2003; Galan et al., 2012).

From Figure 1, the coefficients proposed by Galan et al. provide a better performance compared to the other sets both in linear dispersion $\left(\varepsilon_{c}\right)$ and, specially, in linear shoaling $\left(\varepsilon_{s}\right)$. The G12 coefficients in Table 1 were found so as to improve the performance for any $\kappa$ up to $\kappa_{\max }=5$ obtaining $\left|\varepsilon_{c}, \varepsilon_{s}\right|<$ $0.3 \%$, and the corresponding sets for wider ranges (i.e., up to deeper waters) such as $\kappa_{\max }=10$ or $\kappa_{\max }=20$ were also provided.

The above results have been here slightly improved, as shown in Table 2 for different values of $\kappa_{\max }$. In a problem where, e.g., the maximum values 


\begin{tabular}{lrrrrr}
\hline \hline & $\kappa_{\max }=5$ & $\kappa_{\max }=10$ & $\kappa_{\max }=20$ & $\kappa_{\max }=40$ & $\kappa_{\max }=60$ \\
\hline$\alpha$ & -0.55247 & -0.59441 & -0.59412 & -0.58723 & -0.57057 \\
$\delta$ & -0.01597 & -0.05730 & -0.03277 & -0.02044 & -0.02249 \\
$\gamma$ & -0.00014 & -0.03277 & -0.00407 & -0.00176 & -0.00181 \\
$\delta_{h}$ & -0.09701 & 0.09615 & 0.03143 & 0.01593 & 0.02425 \\
$\gamma_{h}$ & -0.05526 & 0.01070 & 0.00201 & 0.00054 & 0.00073 \\
\hline$\varepsilon_{c}=\varepsilon_{s}$ & $0.170 \%$ & $1.60 \%$ & $3.72 \%$ & $7.46 \%$ & $16.8 \%$ \\
\hline \hline
\end{tabular}

Table 2: Constant coefficients and errors for different $\kappa_{\max }$.

of $\kappa$ are expected to be above 10 and below 20 , the coefficients for $\kappa_{\max }=20$ should be used: in that case, the errors in wave celerity and shoaling will be below $3.72 \%$.

From Table 2, the wider the range (i.e., the deeper waters we consider), the higher the error. This is a natural consequence of the perturbative nature of the BTEs. By construction, using constant coefficients in the BTEs, from equation (5) one gets $c \propto \sqrt{g h}$ as $k h$ increases (deep waters), so that one could never obtain the desired result $c=g / \omega$ provided by the Airy theory in deep waters. To circumvent this problem, we will consider here that the coefficients are functions of $h$.

\section{Coefficients functions of water depth $h$}

Here we will consider that the coefficients are functions of the water depth $h$. Thinking in a dimensional way, the coefficients $\alpha, \delta, \gamma, \delta_{h}$ and $\gamma_{h}$ will be functions of gravity $g$, local water depth $h$ and the limits of the angular frequencies in deep waters, $\omega_{\min }$ and $\omega_{\max }$. Applying dimensional analysis, 
and

$$
\frac{\partial u_{\alpha}}{\partial t}+g \frac{\partial \eta}{\partial x}+h^{2}\left[g l_{3} \frac{\partial^{3} \eta}{\partial x^{3}}+l_{4} \frac{\partial^{3} u_{\alpha}}{\partial t \partial x^{2}}\right]+h \frac{\partial h}{\partial x}\left[g s_{3} \frac{\partial^{2} \eta}{\partial x^{2}}+s_{4} \frac{\partial^{2} u_{\alpha}}{\partial t \partial x}\right]=0
$$


where $l_{j}$ and $s_{j}$ are functions of $\alpha, \delta, \gamma, \delta_{h}$ and $\gamma_{h}$. In equation (9a), the term

$$
(\alpha+1) \frac{\partial \alpha}{\partial x} h^{3} \frac{\partial^{2} u_{\alpha}}{\partial x^{2}}=\frac{\partial h}{\partial x}(\alpha+1) \beta_{\alpha} h^{2} \frac{\partial^{2} u_{\alpha}}{\partial x^{2}}, \quad \beta_{\alpha} \equiv h \frac{\partial \alpha}{\partial h},
$$

is new and would cancel if $\alpha$ was constant. This term has the same structure than that corresponding to $s_{2}$ in equation (9a), is order $\mathcal{O}(\partial h / \partial x)$ and affects only the shoaling. The analysis of the linear dispersion and shoaling can be done following the usual procedures, and it is avoided here for clarity in the presentation. From this analysis one gets that the linear dispersion is not affected by the derivatives of the coefficients, so that equation (5) remains valid. Further, the shoaling analysis, and in particular the shoaling gradient $\alpha_{\eta, \text { bte }}$, is affected by $\beta_{\alpha}, \beta_{\delta}$ and $\beta_{\gamma}$, which are defined as

$$
\beta_{a} \equiv h \frac{\partial a}{\partial h}
$$

which happen to be order one.

\section{An analytical approach}

Consider first the deep-water propagation of monochromatic waves with an angular frequency $\omega=\omega_{0}$. In deep waters nonlinear effects are negligible and, hence, monochromatic waves remain monochromatic. In fact, the main feature to be captured by any model equations are wave celerity and amplitude.

Equation (5), which, as mentioned, remains valid for variable coefficients, and equation (6) can be understood as $k_{\mathrm{bte}}=f_{\mathrm{bte}}(\alpha, \delta, \gamma, g, h, \omega)$ and $k_{\text {Airy }}=$ $f_{\text {Airy }}(g, h, \omega)$. Therefore, imposing the linear dispersion to be exact, i.e., $c_{\mathrm{bte}}=c_{\text {Airy }}$, which is equivalent to impose $k_{\mathrm{bte}}=k_{\text {Airy }}$, gives the condition 


$$
\left\{f_{c} \equiv\right\} f_{\text {bte }}\left(\alpha, \delta, \gamma, g, h, \omega=\omega_{0}\right)-f_{\text {Airy }}\left(g, h, \omega=\omega_{0}\right)=0 .
$$

For arbitrary values of $g, h$ and $\omega_{0}$, the above condition can be satisfied in an infinite number of ways since we have three free coefficients. However, considering, e.g., $\delta=\gamma=0$ we can obtain $\alpha$ (or $c_{\alpha}$ ) biunivocally. Recalling that $d_{\alpha}=c_{\alpha}+1 / 3$, we get

$$
c_{\alpha}=\frac{k_{0} h-\left(k_{0} h\right)^{3} / 3-\tanh \left(k_{0} h\right)}{\left(k_{0} h\right)^{2}\left(k_{0} h-\tanh \left(k_{0} h\right)\right)},
$$

where $k_{0} h$ is obtained from $\kappa_{0} \equiv \omega_{0}^{2} h / g$ since $\kappa_{0}=k_{0} h \tanh \left(k_{0} h\right)$. From $c_{\alpha}$ we recover $\alpha$ as $\alpha=-1+\sqrt{1+2 c_{\alpha}}$.

\subsection{Linear dispersion in deep waters}

The above condition (11) was already obtained by Lee et al. (2003) departing from BTEs with only one free parameter $(\alpha$, since $\delta=\gamma=0$ are not present in their approach). Taking advantage of the fact that we have three free coefficients for linear dispersion $(\alpha, \delta$ and $\gamma)$ we will now improve the dispersion performance in a neighbourhood of $\omega=\omega_{0}$. Instead of imposing $f_{c}=0$, in order to improve the performance around $\omega_{0}$ (and to increase the number of equations up to the number of unknowns, three) we consider here

$$
f_{c}\left(\omega=\omega_{0}\right)=\frac{d f_{c}}{d \omega}\left(\omega=\omega_{0}\right)=\frac{d^{2} f_{c}}{d \omega^{2}}\left(\omega=\omega_{0}\right)=0 .
$$

In this way we get a system of three equations for our three unknowns $c_{\alpha}$ (i.e., $\left.\alpha\right), \delta$ and $\gamma$. The analytical solutions of the above equations are shown in Appendix A. In fact, there are four different sets of solutions. The first solution, denoted " $+\&+$ " in the appendix, has values similar to those in 
Table 1 for M98 and G12. The other three solutions have shown to present numerical stability problems and are disregarded. In any case, the functions $\alpha, \delta$ and $\gamma$ turn out to be functions of the dimensionless group $\kappa_{0} \equiv \omega_{0}^{2} h / g$ : this fact has been anticipated through dimensional analysis.

The consequences of imposing the conditions (12) are illustrated in Figure 2 for $\omega_{0}=1 \mathrm{~s}^{-1}$ and considering four different water depths $h$-the values of $\alpha$, $\delta$ and $\gamma$ are different at each water depth $h$ since $\kappa_{0}=\omega_{0}^{2} h / g$ changes-. The error $\varepsilon_{c}$ always cancels at $\omega=\omega_{0}$ and, since the first and second derivatives are null, the error is kept small around $\omega_{0}$. In fact, for $h=250 \mathrm{~m}, 500 \mathrm{~m}$ and $1000 \mathrm{~m}$ the errors behave similarly and are below $1 \%$ for $0.83 \mathrm{~s}^{-1} \leqslant \omega \leqslant$ $1.20 \mathrm{~s}^{-1}$. For $h=50 \mathrm{~m}$, i.e., in shallower waters, the error behaves, naturally, better: in this case the error is below $1 \%$ for $0 \leqslant \omega \leqslant 1.32 \mathrm{~s}^{-1}$. The solution " $+\&+"$ in Appendix A is considered to build Figure 2.

Figure 2: Illustration of the consequences of imposing $f_{c}=\partial f_{c} / \partial \omega=\partial^{2} f_{c} / \partial \omega^{2}=0$ at $\omega=\omega_{0}=1 \mathrm{~s}^{-1}$.

can propagate in arbitrary deep waters waves the range $0.71 \omega_{0} \leqslant \omega \leqslant 1.39 \omega_{0}$ 
with error $\varepsilon_{c}<5 \%$, the range $0.83 \omega_{0} \leqslant \omega \leqslant 1.20 \omega_{0}$ with $\varepsilon_{c}<1 \%$ (as already stated), and the range $0.92 \omega_{0} \leqslant \omega \leqslant 1.09 \kappa_{0}$ with $\varepsilon_{c}<0.1 \%$.

Figure 3: Range of application for the coefficients correspondig to a given $\kappa=\kappa_{0}$. The errors are $\varepsilon_{c}$.

For a given range of frequencies $\left[\omega_{\min }, \omega_{\max }\right]$ and a given maximum depth $h$, the value of $\omega_{0}$ that minimizes the error in the range, which is not necessarily the mean value $\left(\omega_{\min }+\omega_{\max }\right) / 2$, can be found. As already mentioned, in shallow waters, as it corresponds to BTEs, all frequencies are well represented. This fact is clear from Figure 3: the range $\omega / \omega_{0}$ increases as $\kappa_{0} \rightarrow 0$. For instance, for $\kappa_{0}=3$ the errors are below only $0.1 \%$ for any $\omega \lesssim 1.27 \omega_{0}$, what is to say for any $\kappa=\omega^{2} h / g \lesssim 1.27^{2} \omega_{0}^{2} h / g=1.61 \kappa_{0} \approx 4.83$.

\subsection{Linear shoaling in deep waters}

For a given frequency $\omega_{0}$, above we have found the values of $\alpha, \delta$ and $\gamma$, functions of $h$, so as to improve the linear dispersion performance around $\omega_{0}$. In fact, it has been seen that $\alpha, \delta$ and $\gamma$ are functions of $\kappa_{0} \equiv \omega_{0}^{2} h / g$.

A similar reasoning is followed to obtain the coefficients $\delta_{h}$ and $\gamma_{h}$ in Appendix B. Now the focus is on shoaling error $\varepsilon_{s}$ as defined in expression (8). Recall that, because we are considering variable coefficients, the shoaling gradient $\alpha_{\eta \text {,bte }}$ depends on $\alpha, \delta, \gamma, \delta_{h}$ and $\gamma_{h}$ but also on $\beta_{\alpha}, \beta_{\delta}$ and $\beta_{\gamma}$, which are known from the the solution of $\alpha, \delta$ and $\gamma$ obtained from the linear dispersion analysis. As shown in Appendix B, there are four sets of solutions for $\delta_{h}$ and $\gamma_{h}$ corresponding to the different sets obtained for $\alpha, \delta$ and $\gamma$ above. The solution of $\delta_{h}$ and $\gamma_{h}$ corresponding to " $+\&+$ ", the only 
one we are interesed in, presents an infinite discontinuity at $\kappa_{0} \approx 4.2$ (Figure B.13) and, therefore, this approach must be abandoned.

\section{A global minimization approach}

The analytical approach above has shown to yield useful results in determining $\alpha, \delta$ and $\gamma$ as functions of $\kappa_{0} \equiv \omega_{0}^{2} h / g$ to improve the linear dispersion performance for frequencies around $\omega_{0}$. However, it gives undesirable results for $\delta_{h}$ and $\gamma_{h}$ as functions of $\omega_{0}^{2} h / g$ when trying to improve the linear shoaling performance. Therefore, the above approach is of use if the only property to be well represented was wave celerity.

A different approach aimed to improve both linear dispersion and shoaling is proposed here. We consider water waves which in deep waters have frequencies within the range $\omega \in\left[\omega_{\min }, \omega_{\max }\right]$ propagating from a water depth $h_{\max }$ to the shore. We will find $\alpha, \delta, \gamma, \delta_{h}$ and $\gamma_{h}$ at $n$ values of $h$ from $h_{\min }$ to $h_{\max }$, given by

$$
h_{j} \equiv h_{\min }+(j-1) \Delta h, \quad j=1, \ldots, n,
$$

with

$$
\Delta h \equiv \frac{h_{\max }-h_{\min }}{n-1},
$$

where the values of $h_{\min }$ and $n$ are discussed later. From the above definitions $h_{1}=h_{\min }$ and $h_{n}=h_{\max }$. For $h \leqslant h_{1}$ the coefficients will be constant and equal to those at $h_{1}$ while for any $h \in\left[h_{j}, h_{j+1}\right]$ with $j<n-1$, we consider linear interpolations of the values at $h_{j}$ and $h_{j+1}$. 
For given values of $h_{\min }, h_{\max }, \omega_{\min }, \omega_{\max }$ and $\Delta h$ we will get the values of the five coefficients at each $h_{j}$ so as to minimize the error

$$
\varepsilon \equiv \max _{0 \leqslant h \leqslant h_{\max }}^{\omega_{\min } \leqslant \omega \leqslant \omega_{\max }}\left\{\left|\varepsilon_{c}\right|,\left|\varepsilon_{s}\right|\right\}
$$

where $h$ can take any value from 0 to $h_{\max }$.

We note that, while $\varepsilon_{c}$ is "local" - i.e., it depends only on the coefficients at the water depth were the error is evaluated-, the error $\varepsilon_{s}$ depends on all five coefficients evaluated at any depth below the local water depth. For this reason, the minimization of the five coefficients at all $h_{j}$ must be performed at once.

Given $h_{\max }$ and $\omega_{\max }$, the maximum value of $\kappa$ is $\kappa_{\max , \max }=\omega_{\max }^{2} h_{\max } / g$. Although any possibility could be chosen, for illustrative purposes we consider $\kappa_{\max , \max }=\{20,40,60,80,100\}$. Besides, we consider $h_{\min }$ so that the minimum value of $\kappa$ at this depth, which is $\omega_{\min }^{2} h_{\min } / g$, equals 4 . In this way we ensure that the coefficients are constant up to, at least, $\kappa=4$.

Finally, $\Delta h=\left(h_{\max }-h_{\min }\right) /(n-1)$ were $n$ is chosen so that

$$
\frac{\kappa_{\max , \max }-4}{n-1}=4, \quad \text { i.e., } \quad n=\frac{\kappa_{\max , \max }}{4} .
$$

According to the dimensional analysis, for a given $\kappa_{\max \text { max }}$, now the coefficients will be functions of

$$
\kappa_{\max , j} \equiv \frac{\omega_{\max }^{2} h_{j}}{g}, \quad \text { and } \quad \varrho \equiv \frac{\omega_{\min }}{\omega_{\max }},
$$

and the error $\varepsilon$ will be a function of $\kappa_{\max \text { max }}$ and $\varrho$.

The results are shown in Table 3. We note that the minimization problem is complex ( $5 n$ unknowns and a non convex objective function $\varepsilon$ ) and the 


\begin{tabular}{lrrr}
\hline \hline & $\varrho=0.8$ & $\varrho=0.9$ & $\varrho=1.0$ \\
\hline$\kappa_{\text {max } \max }=20$ & $3.67 \%$ & $2.67 \%$ & $0.98 \%$ \\
$\kappa_{\max , \max }=40$ & $6.60 \%$ & $4.51 \%$ & $0.98 \%$ \\
$\kappa_{\max , \max }=60$ & $11.2 \%$ & $6.60 \%$ & $2.46 \%$ \\
$\kappa_{\max , \max }=80$ & $12.1 \%$ & $6.91 \%$ & $2.86 \%$ \\
$\kappa_{\max , \max }=100$ & $12.4 \%$ & $6.91 \%$ & $2.86 \%$ \\
\hline \hline
\end{tabular}

Table 3: Errors.

results could probably be further improved. The table presents the errors $\varepsilon=f\left(\kappa_{\max , \max }, \varrho\right):$ the values of the coefficients $\alpha, \delta, \gamma, \delta_{h}$ and $\gamma_{h}$, functions of $\left\{\kappa_{\max , \max }, \kappa_{\max , j}, \varrho\right\}$ can be found at

$$
\text { https://dl.dropbox.com/u/11753471/web/p110315.zip }
$$

The general expectable trends in Table 3 are the same observed in Figure 3. First, the error diminishes as the $\varrho$ decreases, i.e., as the frequency range is diminished. Second, the error increases with $\kappa_{\max , \max }$, but it seems to tend to a finite error as $\kappa_{\max , \max }$ grows.

For each case in Table 3 , the coefficients $\delta_{\epsilon}$ and $\gamma_{\epsilon}$, constant, have been established following the same procedure as that presented in Galan et al. (2012). The results are presented in the above link.

\section{Numerical scheme and results}

The numerical scheme considered to solve the model equations is the one presented by Galan et al. (2012). This scheme uses a fourth order accuracy finite differences discretization in space and a fourth order Runge-Kutta explicit scheme in time. 
In this Section, three numerical examples are shown in order to demonstrate the capabilities of the proposed equations. The first case is the propagation of a bichromatic linear wave train over a submerged shoal in deep waters, the second is the simulation of one the experiments of the Dingemans bar and the third one considers one of the experiments by Trulsen et al. (2012) for irregular and nonlinear wave propagation.

\subsection{Case 1: linear propagation over sloping bathymetry}

A first example is meant to illustrate the linear performance of the equations with variable coefficients in deep waters. We consider the propagation of a wave train composed by the sum of two monochromatic waves with amplitudes $a_{1}=a_{2}=0.1 \mathrm{~m}$ and periods $\mathrm{T}_{1}=6.0 \mathrm{~s}$ and $\mathrm{T}_{2}=6.5 \mathrm{~s}$.

The bathymetry is a shoal given by

$$
h(\mathrm{~m})=h_{\max }-\left(h_{\max }-h_{\min }\right) \exp \left(-\left(\frac{x-x_{c}}{800}\right)^{2}\right),
$$

with $h_{\max }=300 \mathrm{~m}$ and $h_{\min }=150 \mathrm{~m}$ respectively the maximum and minimum depths (see Figure 5, bottom panel). The top of the bump is located at $x=x_{c}=4750 \mathrm{~m}$ and the maximum slope, at $x=x_{c} \pm 800 / \sqrt{2}$, is $\partial h / \partial x \approx$ 0.098. In this case $\omega_{\min }=2 \pi / \mathrm{T}_{2}=0.967 \mathrm{~s}^{-1}$ and $\omega_{\max }=2 \pi / \mathrm{T}_{1}=1.047 \mathrm{~s}^{-1}$ and, hence

$$
\kappa_{\max , \max }=\frac{\omega_{\max }^{2} h_{\max }}{g} \approx 33.54, \quad \varrho=\frac{\omega_{\min }}{\omega_{\max }} \approx 0.923,
$$

and we consider the coefficients corresponding to $\kappa_{\max , \max }=40$ and $\varrho=0.9$, with errors bounded by $4.51 \%$. The coefficients are provided at 10 different 


\begin{tabular}{cccccc}
\hline \hline$\kappa_{\max , j}$ & $h_{j}(\mathrm{~m})$ & $\alpha$ & $\delta$ & $\gamma$ & $\ldots$ \\
\hline 4.94 & 44.18 & -0.580282 & -0.019680 & -0.000789 & $\ldots$ \\
8.83 & 79.03 & -0.579805 & -0.020601 & -0.001293 & $\ldots$ \\
$\vdots$ & $\vdots$ & $\vdots$ & $\vdots$ & $\vdots$ & $\vdots$ \\
36.10 & 322.98 & -0.578831 & -0.020237 & -0.001801 & $\ldots$ \\
40.00 & 357.83 & -0.577795 & -0.020225 & -0.001703 & $\ldots$ \\
\hline \hline
\end{tabular}

Table 4: Coefficient sets to be used depending on the maximum expected $\kappa$.

Figure 4: Linear coefficients for test case 1 , corresponding to $\kappa_{\max \text { max }}=40$ and $\varrho=0.9$ (here expressed as functions of $h_{j}$ )

values of $\kappa_{\max , j}=\omega_{\max }^{2} h_{j} / g$ equally spaced from $\kappa_{\max , 1}=4.94$ to $\kappa_{\max , 10}=$ $\kappa_{\max , \max }=40$. In table 4 some of them are presented as a function of $h_{j}$.

Linear interpolation gives the values of the coefficients at any of the grid points, $x$, imposing as well constant values corresponding to those at $h_{1}$ in points where $h \leqslant h_{1}$. This is shown graphically in Figure 4, where a constant initial length can be localized below $\kappa_{\max }=4.94$ for all the free coefficients.

Table 5 summarizes the errors made in linear dispersion for the two considered frequencies at two discrete points: the first point at the beginning of the domain where the depth is maximum and the second point on the top of the bump. As shown, maximum error is $3.82 \%(\leqslant 4.51 \%)$.

Figure 5 shows the propagation of the two different frequencies throughout the domain obtained by the numerical model together with the analytical envelope for the amplitude obtained by using the linear theory (which gives 


\begin{tabular}{lrr}
\hline \hline wave component & $j=1$ & $j=2$ \\
\hline$a(\mathrm{~m})$ & 0.1 & 0.1 \\
$\mathrm{~T}(\mathrm{~s})$ & 6.0 & 6.5 \\
\hline \multicolumn{2}{c}{ at $h=300 \mathrm{~m}$} \\
\hline$\kappa$ & 33.54 & 28.57 \\
$\lambda_{\text {Airy }}(\mathrm{m})$ & 56.21 & 65.97 \\
$\lambda_{\text {bte }}(\mathrm{m})$ & 55.42 & 65.65 \\
$\varepsilon_{c}$ & $-1.40 \%$ & $-0.47 \%$ \\
\hline & at $h=150 \mathrm{~m}$ \\
\hline$\kappa$ & 16.77 & 14.29 \\
$\lambda_{\text {Airy }}(\mathrm{m})$ & 56.21 & 65.97 \\
$\lambda_{\text {bte }}(\mathrm{m})$ & 58.36 & 65.31 \\
$\varepsilon_{c}$ & $3.82 \%$ & $1.00 \%$ \\
\hline \hline
\end{tabular}

Table 5: Coefficient sets to be used depending on the maximum expected $\kappa$. 
Figure 5: Numerical results (continuous lines) obtained with linear coefficients corresponding to $\kappa_{\max , \max }=40$ and $\varrho=0.9$ and analytical envelope (discontinuous lines) for free surface elevation. Snapshot at time $=1000 \mathrm{~s}$ for the wave component of $\mathrm{T}=6.0 \mathrm{~s}$ (top panel) and for the wave component of $\mathrm{T}=6.5 \mathrm{~s}$ (middle panel). The bathymetry and the generation area is depicted in the bottom panel.

Figure 6: Time history for free surface elevation at two different locations. Numerical results (line) obtained with linear coefficients corresponding to $\kappa_{\max \text { max }}=40$ and $\varrho=0.9$. The analytical solution is displayed with stars.

nearly constant wave amplitude). For the numerical scheme we considered a mesh size of $1 \mathrm{~m}$ and a time step of $0.25 \mathrm{~s}$, satisfying the CFL condition presented in the work by Galan et al. (2012). The numerically propagated amplitude has a maximum error of $0.9 \%$ for the wave with $\mathrm{T}=6.0 \mathrm{~s}$ and $4.31 \%$ for the one with $\mathrm{T}=6.5 \mathrm{~s}$ (nearly unappreciable in the figure).

Figure 6 shows the time history for free surface elevation at two different locations (\#A, with $x=2500 \mathrm{~m}$, and \#B, with $x=x_{c}=4750 \mathrm{~m}$ ), one at the maximum depth and another one at the top of the shoal, compared with the analytical solution (in phase at \#A). The results for linear dispersion (i.e., wave celerity) compare well and are consistent with the expected results.

\subsection{Case 2: non linear propagation over a bar}

A second example is meant to show how the model equations can handle with the nonlinear behaviour of the wave as they reach shallow waters from 
Figure 7: Linear coefficients for test case 2 , corresponding to $\kappa_{\max , \max }=20$ and $\varrho=1.0$.

deep waters. For this purpose we consider the propagation of a monochromatic wave with period $\mathrm{T}=2.857 \mathrm{~s}$ over a constant slope $(\approx 20 / 300)$ from a maximum depth of $20 \mathrm{~m}$ (i.e., $\kappa \approx 9.9)$ to $0.86 \mathrm{~m}(\kappa \approx 0.42)$. At the end of the slope we introduce the bathymetry by Dingemans (1997) in order to compare the experimental results with those measured in laboratory at different control gages. The bathymetry is shown in Figure 8 (top panel), while Dingemans bathymetry is shown as a zoom.

The wave amplitude generated in the experiment of Dingemans (case A) is $\eta_{0}=0.02 \mathrm{~m}$ over the depth of $0.86 \mathrm{~m}$, so that, to propagate from deep water with an adequate amplitude, and based on the linear theory $\left(a^{2} c_{g}=\right.$ constant, being $a$ the wave amplitude and $c_{g}$ the group celerity), we introduce an amplitude $\eta_{0}=0.0205 \mathrm{~m}$ in the generation deep zone.

For this test we have $\omega_{\min }=\omega_{\max }=2 \pi / \mathrm{T}=2.2 \mathrm{~s}^{-1}$ and, as anticipated

$$
\kappa_{\max , \max }=\frac{\omega_{\max }^{2} h_{\max }}{g} \approx 9.86,
$$

so that we will consider the coefficients corresponding to $\kappa_{\max \text { max }}=20$ and $\varrho=1.0$ (monochromatic). Using this set of coefficients the linear dispersion and shoaling errors are below $0.98 \%$, as shown in Table 3 . The values for linear coefficients are shown in Figure 7 while nonlinear coefficients are $\delta_{\epsilon}=$ -0.276780 and $\gamma_{\epsilon}=0.135060$.

Figure 8 shows the time history comparison between numerical results 
Figure 8: Dingemans' experiments (case A). Numerical results (lines) and experimental data (stars) for the normalized free surface elevation.

and experimental data at 8 different gages (from \#1 to \#8). Section \#1 has been used as control section, allowing to synchronize model and experimental time. As shown, the comparison between numerical and experimental results is good for all considered section.

\subsection{Case 3: non linear irregular waves propagation}

Finally, we present a numerical simulation one of the test presented by Trulsen et al. (2012). The laboratory experiments consist on the propagation of irregular waves travelling from a water depth $h_{\max }=0.60 \mathrm{~m}$ to $h_{\min }=$ $0.30 \mathrm{~m}$ through a 6 meter long ramp (1:20). We consider the "case 1 " in the original paper, the most demanding attending to their dispersive conditions. The significant wave height is around $0.06 \mathrm{~m}$ at $h=h_{\max }$, so that nonlinear effects are significant as the water depth decreases.

For the case under consideration, the Figure 9 shows the wave amplitudes corresponding to the angular frequencies composing the incident signal at $h_{\max }=0.6 \mathrm{~m}$. We discretized the continuous signal with 240 frequencies. From the figure $\omega_{\min } / \omega_{\max } \approx 0.1 \ll 0.8$ and, therefore, the experiments are beyond the scope of the analysis for variable coefficients. The closest set of coefficients would be those for $\varrho=0.8$ and $\kappa_{\max , \max }=20$.

Figure 10 shows the errors at $h=0.6 \mathrm{~m}$ in linear dispersion and shoaling using constant coefficients (those for $h \leqslant h_{1}$ ) corresponding to $\varrho=0.8$ and $\kappa_{\max , \max }=20$, which are $\alpha=-0.590334, \delta=-0.032415, \delta_{h}=0.031415$, 
Figure 9: Frequencies and amplitudes of each harmonic composing the incident signal.

Figure 10: Linear dispersion and shoaling errors as a function of $\omega$ for $h=0.6 \mathrm{~m}$ and the constant coefficients correspoding to $\varrho=0.8$ and $\kappa_{\max , \max }=20$.

$\gamma=-0.004324$ and $\gamma_{h}=0.001212$. As depectid in the figure, the errors are $\lesssim 4 \%$ for the whole range of frequencies. Obviously, the results are better at $h=0.3 \mathrm{~m}$.

Figure 11 shows the comparion of the spectra at the different gages (experimental and computed). The numerical results show fair agreement with the experimental data. Besides the above errors above $4 \%$, it is to mention that in this experiment strong nonlinearities and strong dispersive conditions coincide. This is beyond the scope of low order Boussinesq-type equations, which can handle strong nonlinearities in weakly dispersive conditions. Also, according to Tucker and Pitt (2001), a statistical instability exists due to wave density spectrum estimation from a finite record (wave density spectrum has been estimated by scanning), so the estimated spectrum could show differences when compared with the real one.

Figure 11: Normalized wave density spectrum at the 8 different gages. Full line represent data from Trulsen et al. (2012) (case 1) and points are results obtained by the proposed model propagating the incident spectrum. 


\section{Concluding remarks}

The possibility of using variable coefficients (functions of the water depth) in enhanced Boussinesq-type equations has been studied and presented. An analytical approach is disregarded since it has shown to give infinite discontinuities in the solutions. Alternatively, the coefficients are numerically found so as to optimize the linear performance in terms of dispersion and shoaling over mild slopes. The results are presented in dimensionless general form.

The performance of the model is determined by the ratio between the minimum to maximum deep water wave angular frequencies, $\varrho \equiv \omega_{\min } / \omega_{\max }$, and a $k h$-type number, $\kappa_{\max \text { max }}$. The results are particularly interesting for

$\varrho \lesssim 1$, i.e., for narrow banded swells approaching to the coast. For these conditions, the wave can be propagated with small errors in linear dispersion and shoaling up to very deep waters. The theoretical results are supported by numerical simulations compared to analytical and experimental results.

\section{Acknowledgement}

Authors would like to thank support from MICINN through Project 445 CGL2011-22964. G. Simarro and R. Minguez are supported by the Spanish government through the "Ramón y Cajal" program.

\section{Appendix A. Dispersion: coefficients $\alpha, \delta$ and $\gamma$}

For given $g, h$ and $\omega_{0}$, the solution of equations (12) is 


$$
\begin{aligned}
& \delta=\left(\varrho_{1} \pm \sqrt{\varrho_{1}^{2}-4 \varrho_{2}}\right) / 2, \\
& \gamma=\left(\varrho_{1}+1 / 3 \pm \sqrt{\varrho_{1}^{2}+1 / 9+2 \varrho_{1} / 3-4 \varrho_{3}}\right) / 2
\end{aligned}
$$

427

and $c_{\alpha}=\varrho_{1}-\gamma-\delta$ so that, since $c_{\alpha} \equiv \alpha^{2} / 2+\alpha$, we can recover the coefficient $\alpha$ as $\alpha=-1+\sqrt{1+2 c_{\alpha}}$. Above

$$
\varrho_{1}=\frac{n_{1}}{3 \xi_{0}^{2} d}, \quad \varrho_{2}=\frac{n_{2}}{3 \xi_{0}^{4} d}, \quad \varrho_{3}=\frac{n_{3}}{3 \xi_{0}^{5} d},
$$

with $\xi_{0}$ verifying $\xi_{0} \tanh \xi_{0}=\kappa_{0}\left\{\equiv \omega_{0}^{2} h / g\right\}$ and

$$
\begin{aligned}
n_{1} & \equiv 6\left\{2 s_{0}^{2} \xi_{0}^{2}+5\right\} t_{0}^{2}+\left\{2 s_{0}^{2} \xi_{0}^{4}+\left(-12 s_{0}^{2}+1\right) \xi_{0}^{2}-6\left(7 s_{0}^{2}+3\right)\right\} \xi_{0} t_{0}+ \\
& +\left\{-s_{0}^{2} \xi_{0}^{2}+6\left(2 s_{0}^{4}+3 s_{0}^{2}\right)\right\} \xi_{0}^{2}, \\
n_{2} & \equiv 3\left\{2 s_{0}^{2} \xi_{0}^{2}+15\right\} t_{0}^{2}+\left\{2 s_{0}^{2} \xi_{0}^{4}-3\left(2 s_{0}^{2}+1\right) \xi_{0}^{2}-9\left(3 s_{0}^{2}+7\right)\right\} \xi_{0} t_{0}+ \\
& +\left\{3 s_{0}^{2} \xi_{0}^{2}+3\left(2 s_{0}^{4}+5 s_{0}^{2}+8\right)\right\} \xi_{0}^{2}, \\
n_{3} & \equiv 24 t_{0}^{3}+\left\{2 s_{0}^{2} \xi_{0}^{4}+\left(6 s_{0}^{2}-1\right) \xi_{0}^{2}-27\right\} \xi_{0} t_{0}^{2}+ \\
& +\left\{-7 s_{0}^{2} \xi_{0}^{2}+9\left(-3 s_{0}^{2}+1\right)\right\} \xi_{0}^{2} t_{0}+\left\{2 s_{0}^{4} \xi_{0}^{2}+3\left(2 s_{0}^{4}+5 s_{0}^{2}\right)\right\} \xi_{0}^{3}, \\
d & \equiv\left\{2 s_{0}^{2} \xi_{0}^{2}+3\right\} t_{0}^{2}-\left\{2 s_{0}^{2} \xi_{0}^{2}+\left(5 s_{0}^{2}+1\right)\right\} \xi_{0} t_{0}+\left\{2 s_{0}^{4}+s_{0}^{2}\right\} \xi_{0}^{2},
\end{aligned}
$$

with $s_{0} \equiv \operatorname{sech} \xi_{0}$ and $t_{0} \equiv \tanh \xi_{0}$.

The coefficients $\alpha, \delta$ and $\gamma$ are, thus, functions of $\kappa_{0} \equiv \omega_{0}^{2} h / g$. As $\kappa_{0} \rightarrow 0$, $\kappa_{0} \rightarrow \xi_{0}^{2}$ and $\varrho_{1} \rightarrow-4 / 9, \varrho_{2} \rightarrow 1 / 63$ and $\varrho_{3} \rightarrow 1 / 945$, so that we recover the Padé [4/4] approximation (Madsen and Schaffer, 1998; Gobbi et al., 2000). In equations (A.1), there are four possible combinations depending on the signs, equivalent to the four possible solutions discussed by Madsen and Schaffer (1998). 
Figure A.12 shows the three functions $\alpha, \delta$ and $\gamma$ in all four cases. The coefficients are so that $\beta_{\alpha} \equiv h \partial \alpha / \partial h=\kappa_{0} \partial \alpha / \partial \kappa_{0}, \beta_{\delta}$ and $\beta_{\gamma}$ are small. For "+\&+" the values are similar to the values by M98 and G12 in Table 1. However, all four solutions give the same results in terms of linear dispersion.

Figure A.12: Coefficients $\alpha$ (full line), $\delta$ (dashed line), $\gamma$ (dash-dotted line), which are functions of $\kappa_{0} \equiv \omega_{0}^{2} h / g$, depending on the signs considered in equations (A.1). For instance, the case " $+\&-$ " results from considering "+" in equation (A.1a) and "_" in equation (A.1b).

\section{Appendix B. Shoaling: coefficients $\delta_{h}$ and $\gamma_{h}$}

For given $\omega$ and $h$, the error in shoaling is (Chen and Liu, 1995)

$$
\varepsilon_{s}=\exp \left(\int_{0}^{h} \frac{\alpha_{\eta, \text { Airy }}-\alpha_{\eta, \text { bte }}}{h_{*}} d h_{*}\right)-1
$$

where here $\alpha_{\eta, \text { Airy }}=\alpha_{\eta, \text { Airy }}\left(\omega^{2} h_{*} / g\right)$ and

$$
\alpha_{\eta, \text { bte }}=\alpha_{\eta, \text { bte }}\left(\omega^{2} h_{*} / g, \alpha, \delta, \gamma, \delta_{h}, \gamma_{h}, \beta_{\alpha}, \beta_{\delta}, \beta_{\gamma}\right)
$$

are the shoaling gradients corresponding to Airy and BTEs. In the shoaling gradient $\alpha_{\eta, \text { bte }}$, the $\alpha, \delta, \gamma$ and their corresponding $\beta$ 's are known from Appendix A.

Since we now have two (not three) free coefficients, $\delta_{h}$ and $\gamma_{h}$, we impose the two conditions, equivalent to the conditions (12) in the linear dispersion analysis, 


$$
\varepsilon_{s}\left(\omega=\omega_{0}\right)=\frac{\partial \varepsilon}{\partial \omega}\left(\omega=\omega_{0}\right)=0,
$$

450

at any $h$ to obtain $\delta_{h}$ and $\gamma_{h}$ as a function of $h$ for the $\omega_{0}$ used in the dispersion analysis. Defining $f_{s} \equiv \alpha_{\eta \text {, Airy }}-\alpha_{\eta \text {,bte }}$, the above is equivalent to impose, at any $h$,

$$
\int_{0}^{h} \frac{f_{s}}{h_{*}} d h_{*}=\int_{0}^{h} \frac{\partial f_{s} / \partial \omega}{h_{*}} d h_{*}=0,
$$

always evaluated at $\omega=\omega_{0}$.

Given $\omega_{0}$, consider that we know the values of $\delta_{h}$ and $\gamma_{h}$ to sastisfy the conditions (B.1) up to some given depth $h-\Delta h$ (with $\Delta h$ infinitesimal). Taking into account that equation (B.1) already holds at $h-\Delta h$, imposing it at $h$ is simply

$$
f_{s}\left(h, \omega=\omega_{0}\right)=\frac{\partial f_{s}}{\partial \omega}\left(h, \omega=\omega_{0}\right)=0 .
$$

The above nonlinear system has been solved using Newton's method to obtain $\delta_{h}$ and $\gamma_{h}$ at any $h$ and for a given $\omega_{0}$. Again, the resulting $\delta_{h}$ and $\gamma_{h}$ are functions of $\kappa_{0} \equiv \omega_{0}^{2} h / g$. Depending on the solution considered for $\alpha, \delta$ and $\gamma$ (Figure A.12), Figure B.13 shows the resulting $\delta_{h}$ and $\gamma_{h}$.

Figure B.13: Coefficients $\delta_{h}$ (full line) and $\gamma_{h}$ (dashed line), functions of $\kappa_{0} \equiv \omega_{0}^{2} h / g$, depending on the signs considered in equations (A.1) to obtain $\alpha, \delta$ and $\gamma$.

462 From Figure B.13, the solutions " $+\&+$ " and " $-\&-$ " violate the condition of slow variations. In fact, the have discontinuities to the infinity. 


\section{References}

Agnon, Y., Madsen, P. A., Schaffer, H., 1999. A new approach to high order boussinesq models. Journal of Fluid Mechanics 399, 319-333.

Beji, S., Nadaoka, K., 1999. A spectral model for unidirectional nonlinear wave propagation over arbitrary depths. Coastal Engineering 36, 1-16.

Chen, Y., Liu, P. L.-F., 1995. Modified boussinesq equations and associated parabolic models for waterwave propagation. Journal of Fluid Mechanics $288,351-381$.

Dean, R. G., Dalrymple, R. A., 1984. Water wave mechanics for engineers and scientists. Prentice-Hall, Inc., Englewoods Cliffs, New Jersey 07632.

Dingemans, M. W., 1997. Water wave propagation over uneven bottoms. World Scientific, Singapore.

Galan, A., Simarro, G., Orfila, A., Simarro, J. P., Liu, P. L.-F., 2012. A fully nonlinear model for water wave propagation from deep to shallow waters. Journal of Waterway, Port, Coast and Ocean Engineering, in press.

Gobbi, M. F., Kirby, J. T., Wei, G., 2000. A fully nonlinear boussinesq model for surface waves: part ii. extension to $o(k h)^{4}$. Journal of Fluid Mechanics 405, 182-210.

Green, A. E., Naghdi, P. M., 1976. A derivation of equations for wave propagation in water of variable depth. Journal of Fluid Mechanics 78, 237-246. 
Karambas, T. V., Memos, C. D., 2009. Boussinesq model for weakly nonlinear fully dispersive water waves. Journal of Waterway, Port, Coastal, and Ocean Engineering 135(5), 187-199.

Lee, C., Cho, Y.-S., Yoon, S. B., 2003. A note on linear dispersion and shoaling properties in extended boussinesq equations. Ocean Engineering 30, 1849-1867.

Lynett, P., Liu, P. L.-F., 2004. A two layer approach to wave modeling. The Royal Society London, A 460, 2637-2669.

Madsen, P. A., Bingham, H. B., Liu, H., 2002. A new boussinesq method for fully nonlinear waves from shallow to deep water. Journal of Fluid Mechanics 462, 1-30.

Madsen, P. A., Bingham, H. B., Schaffer, H. A., 2003. Boussinesq-type formulations for fully nonlinear and extremely dispersive water waves: derivation and analysis. Royal Society of London A 459, 1075-1104.

Madsen, P. A., Schaffer, H. A., 1998. Higher-order boussinesq-type equations for surface gravity waves: derivation and analysis. Phil. Trans. Royal Society of London A 356, 3123-3184.

Madsen, P. A., Sorensen, O. R., 1992. A new form of the boussinesq equations with improved linear dispersion characteristics. part 2: A slowly-varying bathymetry. Coastal Engineering 18, 183-204.

Nwogu, O., 1993. Alternative form of boussinesq equations for nearshore wave 
505

506

507

508

509

510

511

512

513

514

515

propagation. Journal of Waterway, Port, Coastal and Ocean Engineering $119(6), 618-638$.

Peregrine, D. H., 1967. Long waves on a beach. Journal of Fluid Mechanics $27,815-827$.

Trulsen, K., Zeng, H., Gramstad, O., 2012. Laboratory evidence of freak waves provoked by non-uniform bathymetry. Physics of Fluids 9.

Tucker, M. J., Pitt, E. G., 2001. Waves in Ocean Engineering. Elsevier Science, Amsterdam.

Wei, G., Kirby, J. T., Grilli, S. T., Subramnya, R., 1995. A fully nonlinear boussinesq model for surface waves 1. highly nonlinear unsteady waves. Journal of Fluid Mechanics 294, 71-92. 